\title{
A New Snake Model for Pupil Localization Using Orthogonal Polynomials Transform
}

\author{
R. Krishnamoorthy and D. Indradevi
}

\begin{abstract}
In this paper a new approach for representing and evolving deformable contour or snake model to accurately detect pupil boundary for improving the performance of iris recognition systems is proposed. The proposed model extracts the boundary with computationally efficient Laplacian of Guassian (LoG) mask. The LoG mask is obtained from the set of polynomial basis operators derived from Orthogonal Polynomials Transform. Two types of controlling force models, introduced as internal and external forces are designed to properly activate the contour and locate it over the pupil boundary. The internal forces are designed to smooth the contour as well as to keep it close to a boundary by pushing the contour vertices towards the boundary of interest. The external forces are responsible for pulling the contour vertices towards the pupil boundary and the contour is deformed into a new shape in response to the effective force. A revised contour estimate of the selected boundary based on the execution of the contour tracing algorithm is generated. The results are highly encouraging to capture the contour of non-circular shaped pupil of iris. Experimental results on the CASIA v1.0 iris database demonstrate that the proposed snake model outperforms in both accuracy and speed.
\end{abstract}

Index Terms-Active contour, orthogonal polynomials, iris segmentation.

\section{INTRODUCTION}

Iris based biometric recognition outperforms other biometric methods in terms of accuracy. One of the most important steps in iris recognition systems is iris localization, which is related to the detection of the exact location and contour of iris in an eye image. Obviously, the performance of the identification system is closely related to the precision of the iris localization step [1-2]. For iris localization, most segmentation methods reported in the literature assumed that the inner and outer boundaries of the iris were circular. The initial stage of iris recognition system deals with iris segmentation. The best known iris segmentation algorithm is perhaps due to the work of Daugman [3]. He proposed the Integro-differential operator which assumes that pupil and limbus have circular shapes and searches for large circular variations in the image to detect these boundaries. The Hough transform has been another successful alternative for localizing pupil in an image [4]. In this method, a binary edge map is created by thresholding the magnitude of image intensity gradient and the desired boundaries are detected by maximizing the defined Hough transform. Trucco et al. [5] tried to find the optimal circle parameters of the iris

Manuscript received August 2, 2012; revised October 18, 2012.

The authors are with the Department of CSE, Anna University of Technology, Tiruchirappalli, India (e-mail: rkrish26@hotmail.com). boundaries using simulated annealing algorithm. The results of the circle based method are sensitive to the image rotation, particularly if the angular rotation of the input image is more than 10 degrees.

Due to shape deformation within the object classes of iris, a number of segmentation techniques are reported to extract pupil boundary such as region based, knowledge based and deformable contour model. One of the most popular and successful approaches has been the deformable contour due to its ability to accurately recover the shape of pupil boundary. Deformable contour involves the formulation of a propagating interface, which is a closed curve in 2-D or a closed surface in 3-D, and moves under a speed function determined by local, global and independent properties. Existing deformable contours are parametric deformable model and geometric models. Parametric deformable models originating from the active contour model, introduced by Kass et al.[6] that explicitly represent the interface as parameterized contour in a Lagrangian framework and Geometric deformable models originating from the Level Set method introduced by Osher and Sethian[7] are popular segmentation techniques. The goal of the contour model is to effectively extract the boundary of the pupil without any circularity constraint. N.J.Ritter et al. [8] proposed a method based on the concept of circular active contour, which searches for pupil and limbus boundaries by finding the equilibrium of two defined forces: internal forces and external forces. Sun et al. [9] proposed iris localization with low frequency information of the wavelet transform and also localize iris with a different integral operator. Arvacheh et al. [10] and Abhyankar et al. [11] proposed active contour models to localize the pupillary and limbic boundaries using Level set. Daugman's recent work [12] also uses an active contour model for more precise boundary detection.

The performances of the traditional snake model depend heavily on the location of the initial contour, and modulation of the weigh coefficients. It is not flexible to use because many complex methods are adopted to evolve contours. In order to reduce the influence of the location of initial contour to the final result, the snake formulation can be extended to include these challenges and to recover irregular boundary of pupil based on deformable contour. Hence in this paper, we propose a new deformable contour that uses a physics-based system to model the pupil segmentation [13-14], to achieve very good localization results and hence can be used to enhance the performance of any iris recognition system, since all the downstream processes strongly depend on successful localization. In this approach there is no need for approximation of the spatial derivatives by finite differences. The internal energy is performed entirely in the frequency domain and is significantly faster when compared to the 
solution in spatial domain.

The remainder of this paper is organized as follows: In Section II class of orthogonal polynomials for obtaining point-spread operator for different sizes and the generating formula for the set of polynomial functions are presented. The proposed frame work for pupil localization is presented in Section III. Experimental results and comparison with existing techniques are presented in Section IV and Section V concludes the paper.

\section{ORTHOGONAL POLYNOMIALS TRANSFORM}

In this section we describe the proposed orthogonal polynomials for analyzing the pupil localization. The orthogonal polynomials that have already been well established for image compression [15] are extended in this proposed active contour model.

In order to find the boundary of a pupil, a linear 2-D image formation system is considered around a cartesian coordinate separable, blurring, point spread operator in which the image I results in thesuperposition of the point source of impulse weighted by the value of the object function $f$. Expressing the object function $\mathrm{f}$ in terms of derivatives of the image function I relative to its cartesian coordinates is very useful for analyzing the low level features of the image. The point spread function $\mathrm{M}(x, y)$ can be considered to be real valued function defined for $(x, y) \in X \times Y$, where $X$ and $Y$ are ordered subsets for real values. In case of gray-level image of $\operatorname{size}(n \times$ $n$ ) where $X$ (rows) and $Y$ (columns) consists of a finite set, which for convenience can be labeled as $\{0,1, \ldots . n-1\}$, the function $(x, y)$ reduces to a sequence of functions.

$$
M(i t)=u_{i}(t), \quad i, t=0,1, \ldots \ldots, n-1
$$

The linear two dimensional transformation can be defined by the point spread operator $M(x, y) M(I, t)=u_{i}(t)$, as:

$$
\beta(\varepsilon, \eta)=\int_{x \varepsilon X} \int_{y \varepsilon Y} M(\varepsilon, x) M(\eta, y) I(x, y) d x d y
$$

Considering both $X$ and $Y$ to be a finite set of values $\{0,1$, $2, \ldots n-1\}$, equation(2) can be written in matrix notation as follows.

$$
\left|\beta_{i j}^{\prime}\right|=|M| \otimes|M|^{T}|I|
$$

where $\otimes$ is the outer product and $|I|$ is the image and $\left|\beta_{i j}^{\prime}\right|$ are the coefficients of transformation and the point spread operator $|M|$ is

$$
|M|=\left|\begin{array}{rrrr}
u_{0}\left(x_{0}\right) & u_{1}\left(x_{1}\right) & \cdots & u_{n-1}\left(x_{1}\right) \\
u_{0}\left(x_{1}\right) & u_{1}\left(x_{1}\right) & \cdots & u_{n-1}\left(x_{1}\right) \\
& \vdots & & \\
u_{0}\left(x_{n-1}\right) & u_{1}\left(x_{n-1}\right) & \cdots & u_{n-1}\left(x_{n-1}\right)
\end{array}\right|
$$

We consider the set of orthogonal polynomials $u_{0}(t)$, $u_{1}(t), \ldots . u_{n-1}(t)$ of degrees $0,1,2, \ldots . n-1$, respectively to construct the polynomial operators of different sizes from equation(4) for $n \geq 2$ and $t_{i}=i$. The generating formula for the polynomials is as follows,

$$
\begin{aligned}
& u_{i+1}(t)=(t-\mu) u_{i}(t)-b_{i}(n) u_{i-1}(t) \text { for }(i>1), \\
& u_{i}(t)=t-\mu \text { and } u_{\mathrm{o}}(t)=1
\end{aligned}
$$

where

$$
b_{i}(n)=\frac{\left\langle u_{i}, u_{i}\right\rangle}{\left\langle u_{i-1}, u_{i-1}\right\rangle}=\frac{\sum_{i=1}^{n} u_{i}^{2}(t)}{\sum_{i=1}^{n} u_{i-1}^{2}(t)}
$$

and

$$
\mu=\frac{1}{n} \sum_{t=1}^{n} t
$$

Considering the range of values of to be $t_{i}=i, i=1,2$, $3, \ldots n$, we get

$$
b_{i}(n)=\frac{i^{2}\left(n^{2}-i^{2}\right)}{4\left(4 i^{2}-1\right)}, \quad \mu=\frac{1}{n} \sum_{t=1}^{n} t=\frac{n+1}{2}
$$

\section{A. Orthogonal Polynomials Basis}

For the computational simplicity, the finite Cartesian coordinate set $X, Y$ is labeled as $\{1,2$, and 3$\}$. The point spread operator in equation (3) that defines the linear orthogonal transformation for the image can be obtained as $|M| \otimes|M|$, where $|M|$ can be computed and scaled from equation(4) as follows.

$$
|M|=\left|\begin{array}{lll}
u_{0}\left(x_{0}\right) & u_{1}\left(x_{0}\right) & u_{2}\left(x_{0}\right) \\
u_{0}\left(x_{1}\right) & u_{1}(x)_{1} & u_{2}\left(x_{1}\right) \\
u_{0}\left(x_{2}\right) & u_{1}\left(x_{2}\right) & u_{2}\left(x_{2}\right)
\end{array}\right|=\left|\begin{array}{ccc}
1 & -1 & 1 \\
1 & 0 & -2 \\
1 & 1 & 1
\end{array}\right|
$$

The set of polynomial basis operators $o_{i j}^{n}(0 \leq i, j \leq n-1)$ can be computed as

$$
O_{i j}=\hat{u}_{i} \otimes \hat{u}_{j}^{t}
$$

where $\hat{u}_{i}$ is the $(t+1)^{\text {st }}$ column vector of $|M|$. It can be shown that a set of $(n \times n)(n \geq 2)$ polynomial operators forms a basis, i.e. it is complete and linearly independent. It is also proved that the orthogonal transformation defined by the orthogonal system $|M|$ is complete. Having designed the Orthogonal Polynomials Transform, the proposed framework for pupil localization is presented in the next section.

\section{PROPOSED FRAMEWORK FOR PUPIL LOCALIZATION}

Assuming that the pupil boundary is non-circular, a rectangular contour is defined within the pupil to detect the pupil boundary with pupil center as the seed point. The contour may evolve from any arbitrary position of pupil and reaches the desired boundary. The proposed Orhtogonal Polynomials Transform (OPT) based deformable model is basically emerged from the traditional active contour model. The segmentation of the arbitrarily shaped pupil is designed with low pass filter in OPT domain and the image force is assigned with the image gradients. The contour region is a 
function of 2 spatial coordinates and is represented by a set of orthogonal polynomials. In this representation, the contour region is considered to be a linear combination of uncorrelated(orthogonal) effects that reperesents the presence of edges and noise. The edges are detected and seperated from noise using second derivative of Laplacian[16]. The Laplacian of Guassian(LoG) $\nabla^{2} \Phi(x, y)$ is given by

$$
\nabla^{2} \Phi(x, y)=-\frac{1}{2 \pi \sigma^{4}}\left(2-\frac{x^{2}+y^{2}}{\sigma^{2}}\right) e^{-\frac{1}{2}\left(\frac{x^{2}+y^{2}}{\sigma^{2}}\right)}
$$

where $\sigma$ is the standard deviation representing the width of the Gaussian distribution and $(x, y)$ represents kernel mask along $x, y$ direction. The LoG operator is represented in terms of the proposed polynomial basis operators. The equivalent $\beta_{i j} s$ for $\operatorname{LoG}(3 \times 3)$ operator is

$$
\begin{aligned}
\left|\beta_{i j}\right| & =\left(|M|^{t}|M|\right)^{-1}|M|^{t}|L o G| \\
& =\left|\begin{array}{lll}
0 & 0 & -10.7 \\
0 & 0 & 0 \\
-10.7 & 0 & 20.3
\end{array}\right|
\end{aligned}
$$

Hence the operator LoG is now represented as

$$
-10.7\left(O_{02}^{3}+O_{20}^{3}\right)+20.3 O^{3}
$$

The contour region is convolved with the LoG operator by sliding a window and extract the width of the contour region is same as the width of the operator. After applying the LoG operator, the discontinuties or bending represents the theoretic internal forces that smooth the contour region. To evolve the contour towards the desired boundary using interpolation the governing force $\mathrm{E}$ is computed from the equation (10) as follows:

$$
E=-\nabla \Phi(x, y)
$$

where $E$ is the function of the gradient in the x and y direction which is the spatial coordinates of contour vertex corresponds to the edges in LoG. From E, the time step for the contour is calculated, in order not to cross over possible edges of interest is computed as:

$$
\Delta t^{2}=\frac{h}{E_{\max }}=\frac{h}{\left|\sqrt{E_{x}^{2}+E_{y}^{2}}\right| \max }
$$

where $E_{x}$ and $E_{y}$ are the governing force along $\mathrm{x}$ and $\mathrm{y}$ axes, $E_{\max }$ is the maximum governing force and $\mathrm{h}$ is the grid spacing. The contour motion is achieved by computing the distance $d_{x}$ and $d_{y}$ from equation (13) and (14) and its propagation is further restricted by half grid spacing as:

$$
d_{x}=\frac{E_{x}}{E_{\max }} \frac{h}{2} ; d_{y}=\frac{E_{y}}{E_{\max }} \frac{h}{2}
$$

External forces of the image region are computed that is responsible for guiding the snake toward the boundary of interest using the image gradient potential as follows:

$$
\Phi_{i m g}(x, y)=\alpha \frac{\left|\nabla G_{\sigma}(x, y) * I(x, y)\right|}{\left|\nabla G_{\sigma} * I\right|_{\max }}
$$

where $\alpha$ is a weighting factor ( $\alpha 0 \geq$ ) to adjust the image gradient potential, $\nabla$ is the gradient operator, $\mathrm{G}_{\sigma}(\mathrm{x}, \mathrm{y})$ is a 2-D Gaussian function with standard deviation $\sigma$ and $\mathrm{I}(\mathrm{x}, \mathrm{y})$ is the image region. The image potential in equation (16) is incorporated into governing force by defining the effective force in order to deform the contour to new shapes as:

$$
E_{e f f}=-\nabla \Phi_{e f f}=-\nabla\left(\hat{E}+\Phi_{i m g}\right)
$$

where $\hat{E}$ is the normalized governing force and $\Phi_{i m g}$ is the external force respectively. Boundary of the contour deformation is detected from initial contour by setting initial boolean value to each corresponding pixel in the initial contour based upon the following rules: true if it was inside the initial contour and false if it was outside. True pixels were reset to the position of the new contour during contour deformation. The entire deformation is converged with the following inequality and hence the object of interest is detected.

$$
\frac{\Delta \hat{\mathfrak{R}}_{\text {total }}}{\mathfrak{R}_{\text {total }}} \leq \gamma
$$

where $\hat{\mathfrak{R}}_{\text {total }}$ the total amount of pixels in the overall system and $\mathfrak{R}_{\text {total }}$ is the amount of pixels in the contour. After regressive experiment conducted over several images the selection of $\gamma$ in the range $0.01 \leq \gamma \leq 0.03$ is suggested. The true boundary of ROI is somewhere inside the final propagating contour. The final contour of pupil localization is obtained by computing sub pixel precision and advancing the contour to a real distance by substituting the effective force into (15).

\section{EXPERIMENTS AND RESULTS}

The proposed scheme for pupil localization has been experimented with CASIA V.1 iris datasets. The location of initial contour is drawn with pupil center as seed point (superimposed on original sample image) is presented in fig. 1(b) for the original test image shown in fig. 1(a). The polynomial basis operators are obtained from the Orthogonal Polynomials Transform as given in section II and are used for generating the $(3 \times 3)$ LoG mask as given in equation (12). The contour region is partitioned into $(3 \times 3)$ block size in a sliding window and $(3 \times 3)$ LoG mask is applied to obtain the edge components of contour region. The theoretic internal force are represented from the edge components and the contour motion distance is obtained by calculating the governing force $\mathrm{E}$ from the spatial coordinates of edge components as given in equation (13). The external force is computed with image gradient for different values of . Increasing the value of $\alpha$ increase the effect of external forces and makes the contour to move toward the pupil boundary in a faster pace. The contour is deformed into various shapes and reached the desired pupil boundary with internal and external forces and these results are presented in fig.1(c). The proposed method for pupil localization is tested with different values of $\alpha$ and 
the results are presented in table 1. To evaluate the proposed method quantitatively, the sensitivity ( $\mathrm{SN}$ ) and particularity (KP) parameters are measured as given in [13]. For the $\alpha$ value of 0.5 , the proposed method achieves $94.00 \% \mathrm{SN}$ and $80.00 \% \mathrm{KP}$. Similarly for the $\alpha$ value of 0.8 the proposed method achieves $98.40 \% \mathrm{SN}$ and $89.00 \%$ of KP are obtained. The final contour of the proposed pupil localization method is shown in fig. 1 (d) for $\alpha=0.8$ that accurately extracted the pupil boundary, and it is superimposed over the original image is shown in fig. 1(e). However, $\alpha$ should be smaller than 1.2 in orders to prevent the contour from oscillating around the pupil boundary due to the definition of the external forces. After conducting several experiments, the value of $\alpha$ above 1.2 enhances the noise in the image based on the image gradient in equation (16). Figure 2 shows the result of the another sample image with the proposed method in which the pupil boundary is non-circular.

In order to measure the performance of the proposed method, the pupil boundary is detected using the existing active contour model. For the $\alpha$ value of 0.5 , the active contour method achieves $85.00 \% \mathrm{SN}$ and $94.00 \% \mathrm{KP}$. Similarly for the $\alpha$ value of 0.8 , the active contour model by [9] gives $97.50 \% \mathrm{SN}$ and $96.00 \% \mathrm{KP}$ are obtained using active contour model. The outputs of two sample eye images using active contour model with $\alpha=0.8$ are shown in fig 4 and fig. 5 for normal and rotated eye images respectively. The effectiveness of the proposed algorithm is also measured with accuracy and computation time required for pupil localization. The proposed algorithm achieves $99.5 \%$ accuracy with 1.83 milliseconds (ms) computation time for detecting the pupil area for the sample images and the same is presented in table 2 . These pupil localization performance are compared with existing Daugman[11] and Geodesic [17] methods and the results are incorporated in table 2. From the table, it is evident that, the proposed method achieves more accuracy with lesser computation time as compared with existing techniques.

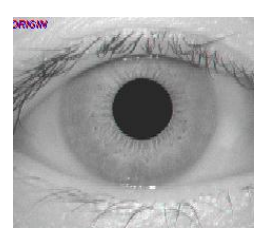

(a)

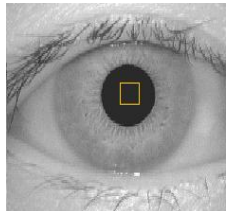

(b)

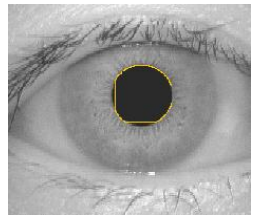

(c)

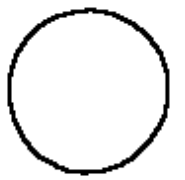

(d)

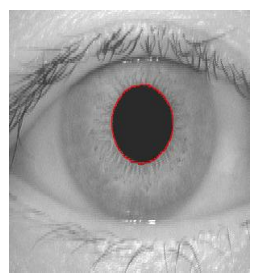

(e)
Fig. 1. Evolution of contour with proposed pupil localization. (a) Original test image (b) Location of initial contour (c) Contour deformation (d) Final pupil contour. (e) pupil localication.

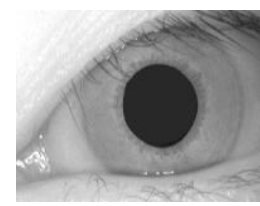

(a)

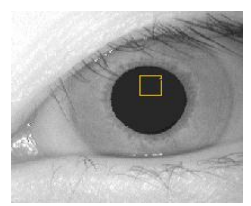

(b)

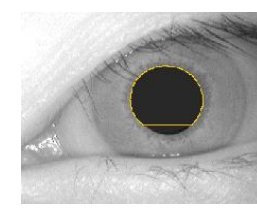

(c)

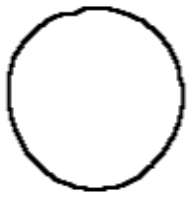

(d)

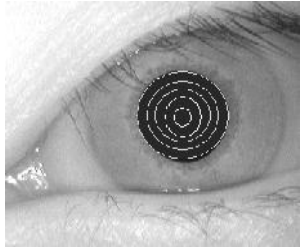

(e)
Fig. 2. Evolution of contour with proposed pupil localization. (a) Original test image (b) Location of initial contour (c) Contour deformation (d) Final pupil contour. (e) pupil localization.

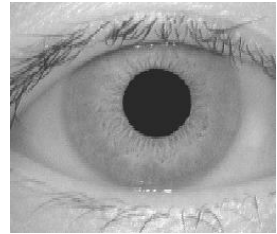

(a)

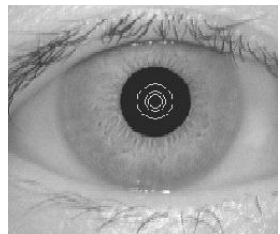

(c)

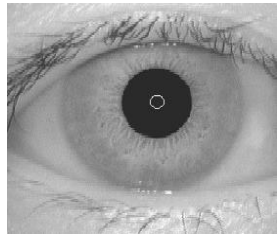

(b)

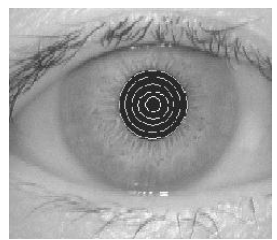

(d)
Fig. 4. Evolution of contour with existing model (a) Normal eyel image (b) Location of initial contour (c) Contour deformation (d) Final pupil contour.

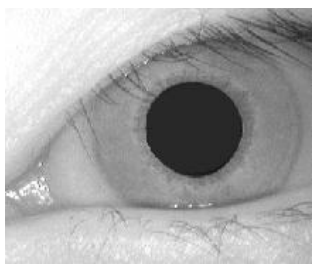

(a)

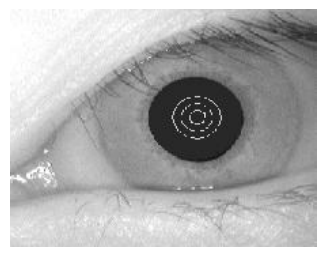

(c)

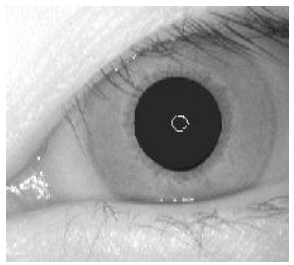

(b)

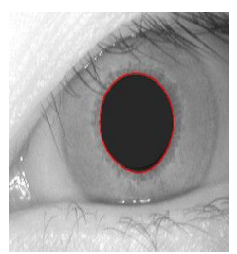

(d)
Fig. 5. Evolution of contour with existing model (a) Origianl eye image (b) Location of initial contour (c) Contour deformation (d) Final pupil contour.

TABLE I: StatistiCAL ANALYSIS OF THE PERFORMANCE MEASURES FOR SENSITIVITY AND PARTICULARITY TEST ON DB USING DIFFERENT VALUES of $\alpha$

\begin{tabular}{|c|c|c|c|c|}
\hline \multirow{2}{*}{$\begin{array}{l}\text { Parameter } \\
\alpha\end{array}$} & \multicolumn{2}{|c|}{ Proposed method } & \multicolumn{2}{|c|}{ Active Contour } \\
\hline & $S N(\%)$ & $K P(\%)$ & $S N(\%)$ & $K P(\%)$ \\
\hline 0.5 & 94.00 & 80.00 & 85.00 & 94.00 \\
\hline 0.6 & 96.00 & 83.00 & 95.00 & 96.00 \\
\hline 0.7 & 98.00 & 84.00 & 97.00 & 94.00 \\
\hline 0.8 & 98.40 & 89.00 & 97.50 & 96.00 \\
\hline
\end{tabular}

TABLE II: SEGMENTATION TIME TO DETECT PUPIL BOUNDARY

\begin{tabular}{|l|l|l|l|}
\hline Method & \multirow{2}{*}{ Accuracy } & $\begin{array}{l}\text { Computation Time } \\
\text { (Milli Seconds) }\end{array}$ \\
\cline { 3 - 4 } & & Sample 1 & Sample 2 \\
\hline Proposed & $99.5 \%$ & 1.83 & 3.89 \\
\hline Daugman & $98.6 \%$ & 3.56 & 4.94 \\
\hline Geodesic & $98.3 \%$ & 4.59 & 6.12 \\
\hline
\end{tabular}




\section{CONCLUSION}

A set of orthogonal polynomials which were used to investigate low level features such as edge and texture, have been utilized to propose a new snake model. The model employs a low-pass filter with OPT as the internal force, which could smooth the contours. The proposed method exhibits some important characteristics such as much less sensitivity to initialization than snakes and only one effective parameter $\alpha$ is used. The proposed approach also addresses the issue of processing iris images where pupil boundaries are not necessarily perfect circle. The experimental results show that the proposed method outperforms the existing methods both in terms of accuracy and computation time.

\section{ACKNOWLEDGMENT}

This work is supported by $\mathrm{R} \& \mathrm{D}$ project sponsored by Ministry of Communication and Information Technology / Department of Information Technology (MIT / DIT) of India. (No. 12(12)/08-ESD).

\section{REFERENCES}

[1] J. G. Daugman, "High condifence visual recognition of Persons by a test of statistical independence," IEEE Transactions on Pattern Analysis and Machine Intelligence, vol.15, pp. 1148- 1161, 1993.

[2] K. W. Bowyer, K. Hollingsworth, and P. J. Flynn, "Image understanding for iris biometrics: a survey," Computer Vision and Image Understanding, vol. 110, pp. 281-307, 2008.

[3] J. Daugman, "How iris recognition works," IEEE Transaction on Circuits and Systems for Video Technology, vol.14, pp. 21-30, 2004.

[4] P. R. Wildes, "Iris recognition in Biomteric Systems: Technology, Design and Performance Evaluation," Spring Verlag, pp. 63-95, 2005.

[5] E. Trucco and M. Razeto, "Robust Iris Location in Close-Up Images of the Eye," Pattern Analysis \& Applications, vol. 8, pp. 247-255, 2005.

[6] M. Kass, A. Witkin, and D.Terzopoulos, "Snake Active contour models," International Journal of Computer Vision, vol.01, no.04, pp. 321-331,1988
[7] S. Osher and J. A. Sethian, "Fronts propagating with curvature dependent speed: algorithm based on hamiltons-jacobi formulation," Journal of Computational Physics vol.79, pp.12- 49, 1988 .

[8] N. J. Ritter and J. R. Cooper, "Locating the iris: A first step to registration and Identification," presented at IASTED International Conference on Signal Processing, 2007.

[9] Z. Sun, Y. Wang, T. Tan, and Y.Wang, "Learning based enhancement model of iris," International Conference on Machine Vision, vol. 46, pp. 153-162, 2003.

[10] E. M. Arvacheh and H. R. Tizhoosh, "Iris Segmentation: Detecting Pupil, Limbus and Eyelids," in Proc. International Conference on Image Processing, pp. 2453-2456, 2006.

[11] A. Abhyankar and S. Schuckers, "Active Shape Models for Effective Iris Segmentation," in Proc. SPIE-Biometric Technology for Human Identification, vol. 6202, pp. 38-48, 2006.

[12] J. Daughman, "New Methods in Iris Recognition," IEEE Transaction on systems, Man and Cybernetics, vol.37, pp. 1167-1175, 2005.

[13] N. Barzegar and M. S. Moin, "A New User Dependent Iris Recognition System Based on an Area Preserving Pointwise Level Set Segmentation Approach," EURASIP Journal on Advances in Signal Processing, vol. 2009, pp. 1-13, 2009.

[14] C. Herng-Hua, D. J. Valentino, G. R. Duckwiler, and A. W. Toga "Segmentation of Brain MR Images using a Charged Fluid Model," IEEE Transaction on Biomedical Engineering, vol. 54, pp. 1798-1813, 2007.

[15] R. Krishnamoorthy and N. Kannan, "A new integer image coding technique based on orthogonal polynomials," Image and vision computing, vol. 27, pp. 996-1006, 2009.

[16] P. Bhattacharya and L. Ganesan, "An orthogonal polynomial based framework for edge detection in 2D monochrome images," Pattern recognition, vol.18, pp. 319-333, 1997.

[17] A. Ross and S.Shah, "Segmenting non-ideak irises using geodesic active contours," in Proceedings of the Biometric Consortium Conference, pp. 1-6, 2006.

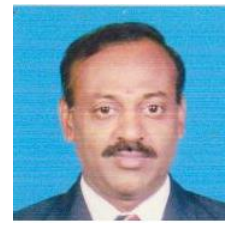

Dr. R. Krishnamoorthy is with computer Science and Engineering, Anna University of Technology Tiruchirappalli. He obtained his Ph.D in Image Processing from the Indian Institute of Technology (IIT), Karaghpur in 1995 and M.Tech in Computer Science and Engineering from the Indian Institute of Technology (IIT), Kanpur, in 1992. His areas of Image Compression, Image Retrieval, Image encryption and authentication Biometrics, Software engineering, Digital Watermarking, Steganography. 